\title{
Compressive Sensing Based Sampling and Reconstruction for Wireless Sensor Array Network
}

\author{
Ming Yin, Kai Yu, and Zhi Wang \\ College of Control Science and Engineering, Zhejiang University, Hangzhou, China \\ Correspondence should be addressed to Zhi Wang; wangzhizju@gmail.com
}

Received 31 March 2016; Accepted 1 August 2016

Academic Editor: Cornel Ioana

Copyright ( 2016 Ming Yin et al. This is an open access article distributed under the Creative Commons Attribution License, which permits unrestricted use, distribution, and reproduction in any medium, provided the original work is properly cited.

\begin{abstract}
For low-power wireless systems, transmission data volume is a key property, which influences the energy cost and time delay of transmission. In this paper, we introduce compressive sensing to propose a compressed sampling and collaborative reconstruction framework, which enables real-time direction of arrival estimation for wireless sensor array network. In sampling part, random compressed sampling and 1-bit sampling are utilized to reduce sample data volume while making little extra requirement for hardware. In reconstruction part, collaborative reconstruction method is proposed by exploiting similar sparsity structure of acoustic signal from nodes in the same array. Simulation results show that proposed framework can reach similar performances as conventional DoA methods while requiring less than $15 \%$ of transmission bandwidth. Also the proposed framework is compared with some data compression algorithms. While simulation results show framework's superior performance, field experiment data from a prototype system is presented to validate the results.
\end{abstract}

\section{Introduction}

The monitoring of noncooperative targets using spatially distributed arrays has been one of the key problems in many applications such as biological acoustic studies [1], gunshot localization [2], and military target tracking [3]. Among all array signal processing methods, direction of arrival (DoA) estimation is an important one for source detection and localization.

In traditional array systems, arrays are connected by wires to a fusion center. In some other systems, like IAASNT [3], sensors in the same array are connected to a local processor. DoA estimation (or other array processing) is done there and only results are transmitted wirelessly to a remote fusion center. But the processors (like DSP) are usually too energyconsuming as well as expensive. These two weaknesses make large-scale wireless deployment and maintenance difficult.

With advances in wireless communication, a few pioneer works $[4,5]$ deployed wireless nodes at spatially dispersed locations to form arrays. Then the AML [4] localization algorithm was used to locate acoustic sources, which validates the feasibility of wireless sensor array network (WSAN).
However the energy and data volume for transmission were not considered. The large amount of raw sensor data needs to be communicated via broadband Wi-Fi channels. Allen et al. [6] proposed the VoxNet system, which uses $802.11 \mathrm{~b}$ wireless protocol to transmit data to the fusion center for processing. Even some strategies are used to reduce data volume; the lifetime is only about 8 hours. This huge energy consumption, along with a rather long transmission delay, would largely harm system performance. Aiming at longer independent detection, low-power, lower-rate wireless protocol is indispensable. Thus many works focus on data reduction issue, like [7].

In this work, we also consider a WSAN configuration. Based on compressive sensing (CS) $[8,9]$, we propose a compressed sampling and collaborative reconstruction framework that can achieve obvious data reduction while keeping similar DoA performance. Instead of transmitting all sensor data to the fusion center, random sampling and deep quantization are performed to significantly reduce the amount of data that needs to be transmitted via wireless channel. Also, unlike traditional data compression algorithm, we place major computation burden at the fusion center side, requiring 


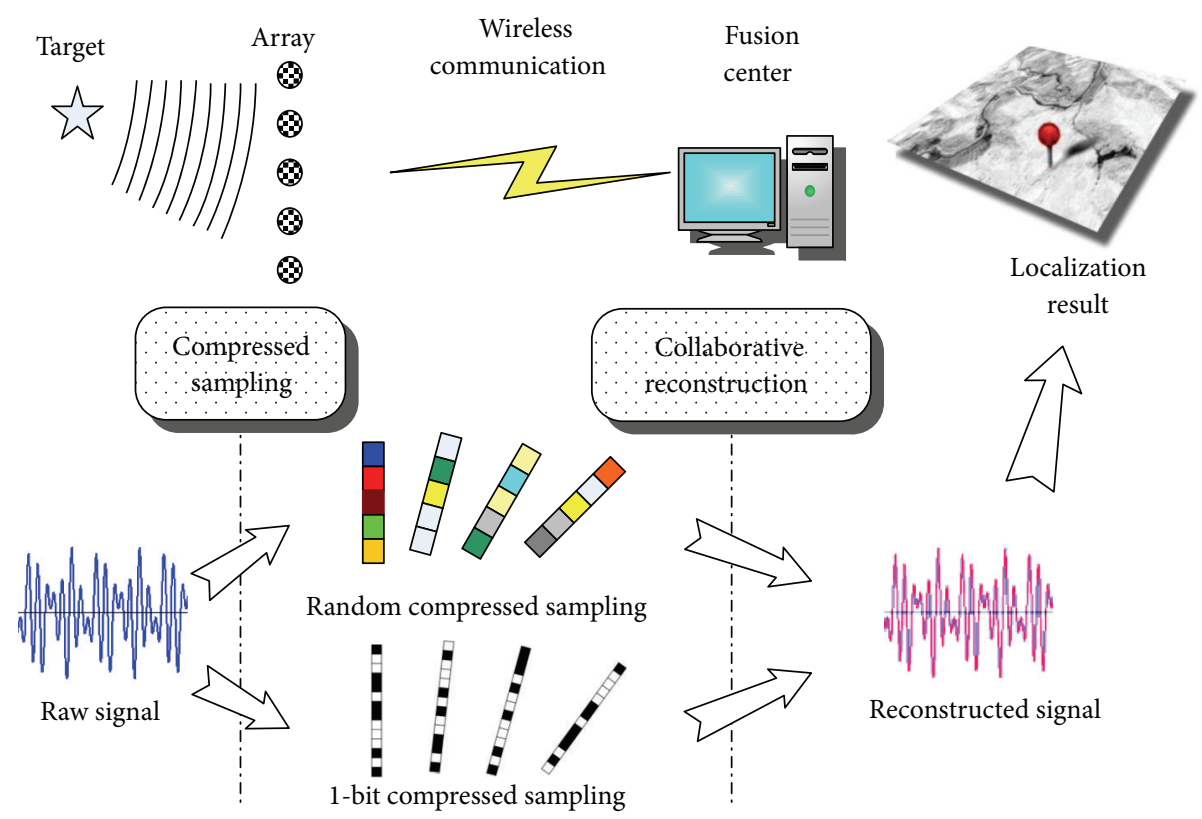

FIGURE 1: Compressed sampling collaborative reconstruction framework.

little computation at individual sensor node. Thus, this framework is very appealing to applications whose resources (energy, transmission, cost, etc.) are limited.

Our framework leverages sparsity in both frequency and space domain. We assume the acoustic signals of sources can be modeled as a sum of few dominant harmonic frequencies. As such, the time series at each sensor has a sparse spectral representation including few dominant spectral lines. Furthermore, sensors belonging to the same array often receive signal with the same frequency components, with only a phase shift due to time difference of arrival, because they are different in distance to the source. Leveraging these two sparsities with compressive sensing theory, we could reduce the data volume to our best.

We employed two methods to exploit the frequency sparsity in sampling the source signals: (1) random sampling of the sensor data and (2) deep quantization of sampled sensor data to the extent that only the sign bit is retained $[10,11]$. To exploit the space domain sparsity, we only apply full reconstruction procedure on sampled data from the reference sensor node. Then the recovered harmonic frequencies' structure will be used to reconstruct the sensor data of remaining sensor nodes using easier methods like least square approach with far fewer data samples than those sampled on the reference node. The procedure of the framework is depicted in Figure 1.

Previously, various works combined sparse reconstruction and array signal processing. Malioutov et al. [12] exploited the sparse distribution characteristic of sources in the angle domain and formulated array processing as a multiple measurement vector (MMV) problem. Baron et al. [13] proposed the joint sparsity model which can be used in sensor arrays. Also many works tried to introduce compressive sensing into wireless sensor network localization problem, like $[14,15]$. However, the problem of data volume for transmission was not solved. Here in our work, our framework provides an acceptable solution to this problem for low-power, low-cost WSAN systems. After successful reconstruction of the array data at the fusion center, either conventional DoA methods such as MUSIC [16] or the sparse representation based approach [12] may be applicable.

Although many differences exist, we compare our framework with modern data compression algorithms to show our data volume reduction ability, including the lossless weighted LZW coding [17], lossy JPEG [18], and MPEG-1 [19]. Also a prototype microphone array platform has been implemented with off-the-shelf devices. Experiment results are presented that convincingly validate our framework's performance. The main contributions of this paper can be summarized as follows:

(a) A novel compressed sampling collaborative reconstruction signal processing framework is proposed.

(b) Random compressed sampling and a newly emerged 1-bit compressed sampling method are employed. Thus sampling data volume is reduced, significantly cutting down the transmission burden.

(c) Leveraging the high correlation of acoustic signals among array elements, a collaborative reconstruction method, works for both random compressed sampling and 1-bit compressive sensing and is presented to drastically reduce computation cost and delay.

(d) Numerical simulation and prototype experiment results are presented to validate the performance of the framework.

This paper is organized as follows: backgrounds of DoA estimation, compressive sensing, and 1-bit compressive 
sensing are simply reviewed in Section 2. Our framework is presented in detail in Sections 3 and 4, about sampling and reconstruction, respectively. Simulation and experiment results are reported in Section 5 along with some analysis. And conclusion is presented in Section 6.

\section{Background}

2.1. Array Processing for DoA. Assume there are $Q$ sources in the sensing field. The $q$ th source emits signal $s_{q}(t)$ consisting of some harmonics:

$$
s_{q}(t)=\sum_{r=1}^{R} b_{q}^{(r)} \sin \left(2 \pi f_{r} t+\varphi_{q}^{(r)}\right)+v_{q}(t),
$$

where $b_{q}^{(r)}$ and $\varphi_{q}^{(r)}$ are the magnitude and the phase of the $r$ th harmonic at frequency $f_{r}$ and $\nu_{q}(t)$ represents other less prominent components. For convenience, one assumes that each $f_{r}$ is an integral multiple of $f_{s} / N\left(f_{r}=k f_{s} / N, k \in\right.$ $0,1, \ldots, N-1)$.

The signal received at the $j$ th sensor is the summation of all signals from the $Q$ sources. That is,

$$
x_{j}(t)=\sum_{q=1}^{Q} s_{q, 0}\left(t-\tau_{q, j}\right)+v_{j}(t),
$$

where the reference sensor node receives $s_{q, 0}(t)$ from the $q$ th acoustic source. $\tau_{q, j}$ is the relative difference of propagation delay of $q$ th source's signal from the reference node to the jth sensor. $v_{j}$ is the additive Gaussian white noise at the $j$ th sensor with zero-mean and variance $\sigma^{2}$.

Figure 2 shows the array model, in which e is the reference orientation, $\boldsymbol{\rho}_{j}$ is sensor position for the $j$ th sensor, and $\boldsymbol{\rho}_{t}$ is target (source) position. In this work, we assume that the diameter of the sensor array is much smaller than the sensorto-target distance. That is,

$$
\max _{0 \leq m, m^{\prime} \leq M-1}\left|\boldsymbol{\rho}_{m}-\boldsymbol{\rho}_{m}^{\prime}\right| \ll \min _{0 \leq j \leq J-1}\left|\boldsymbol{\rho}_{t}-\boldsymbol{\rho}_{j}\right| .
$$

As such, the acoustic wave emitted from the sources can be regarded as plane wave. All sensors in the same array should share the same incidence angle $\theta$ for signal from the same source. It is easy to verify that the relative time delay of the $q$ th source between the $j$ th sensor and the array centroid is

$$
\tau_{q, j}=\frac{1}{c}\left(u_{j} \cos \left(\theta_{q}\right)+y_{j} \sin \left(\theta_{q}\right)\right) .
$$

If we represent $\mathbf{x}_{j}=\left[x_{j}\left(t_{1}\right), x_{j}\left(t_{2}\right), \ldots, x_{j}\left(t_{N}\right)\right]^{T}$ in the discrete Fourier basis $\Psi$, the corresponding Fourier coefficients can be given by

$$
\begin{aligned}
\mathbf{x}_{j} & =\boldsymbol{\Psi} \boldsymbol{\alpha}, \\
\boldsymbol{\alpha} & =\left[x_{j}\left(k_{1}\right), x_{j}\left(k_{2}\right), \ldots, x_{j}\left(k_{N}\right)\right]^{T}, \\
x_{j}(k) & =\sum_{q=1}^{Q} S_{q, 0} e^{-j(2 \pi k / N) \tau_{q, j}}+V_{j}(k) .
\end{aligned}
$$

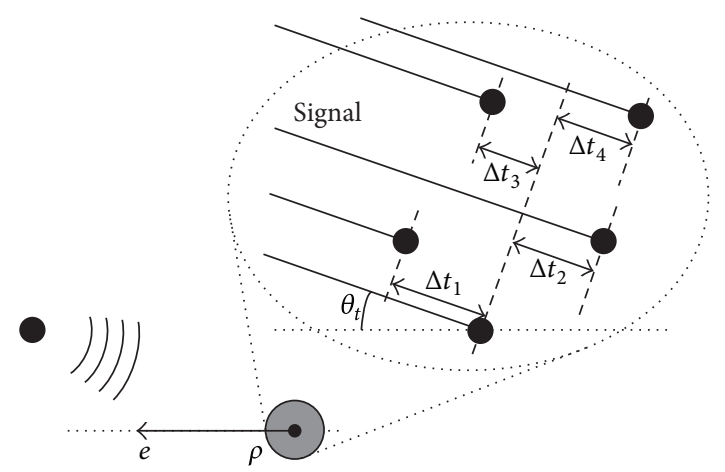

FIgURE 2: DoA estimation model for array.

Consider all the $J$ sensors, the array data spectrum $\mathbf{x}(k)=$ $\left[x_{1}(k), x_{2}(k), \ldots, x_{J}(k)\right]^{T}$ at the $k$ th frequency is given by

$$
\mathbf{x}(k)=\sum_{q=1}^{Q} S_{q, 0} \mathbf{a}(k)+\mathbf{V}(k),
$$

where $\mathbf{a}(k)=\left[e^{-j 2 \pi k \tau_{q, 1} / N}, e^{-j 2 \pi k \tau_{q, 2} / N}, \ldots, e^{-j 2 \pi k \tau_{q, J} / N}\right]^{T}$ is defined as the steering vector corresponding to the $q$ th sources at the $k$ th frequency.

With traditional high resolution DoA spectral estimation, the sample covariance matrix of array data spectrum $\mathbf{x}(k)$ is computed. That is,

$$
\mathbf{R}=\mathbf{x}(k) \mathbf{x}(k)^{T}=\sum_{q=1}^{Q} S_{q, 0}^{2} \mathbf{a}(k) \mathbf{a}(k)^{H}+N \sigma^{2} \mathbf{I} .
$$

Exploiting the low rank structure of the signal covariance matrix $\mathbf{R}-N \sigma^{2} I$, numerous DoA methods, such as ESPRIT and MUSIC [16], have been proposed. Consider eigenvalue decomposition of the matrix $\mathbf{R}$ :

$$
\mathbf{R}=\mathbf{U}_{S} \Sigma_{S} \mathbf{U}_{S}^{H}+\mathbf{U}_{N} \Sigma_{N} \mathbf{U}_{N}^{H},
$$

where $\Sigma_{S}$ and $\Sigma_{N}$ are diagonal matrices consisting of the eigenvalues of $\mathbf{R}$ such that the eigenvalues in $\Sigma_{S}$ are larger than those in $\Sigma_{N}$. Equation (8) implies that the steering vectors $\mathbf{A}(\theta)$ must lie within the span of the signal subspaces $\mathbf{U}_{S}$ and perpendicular to the noise subspace spanned by columns of $\mathbf{U}_{N}$. With the MUSIC method, the DoA of targets are estimated as the peaks of the following function:

$$
P_{\text {MUSIC }}(\theta)=\frac{1}{\mathbf{a}^{H}(\theta) \mathbf{U}_{N} \mathbf{U}_{N}^{H} \mathbf{a}(\theta)} .
$$

2.2. Compressive Sensing. Compressive sensing theory $[8,9]$ states that a sparse signal may be randomly sampled at subNyquist rate and then be reconstructed perfectly. Denote $\boldsymbol{\alpha}$ to be a sparse vector representing the sparse signal, $\mathbf{x}=$ $\Psi \boldsymbol{\alpha}\left(\mathbf{x} \in R^{N}\right)$ to be the original signal, and $\mathbf{y}=\boldsymbol{\Phi}_{\mathbf{x}}$ to be the observed signal. According to compressive sensing theory, the observation matrix $\Phi$ can be chosen to be a Gaussian random matrix or Bernoulli random matrix. 
Given $y$, the CS reconstruction problem is formulated as a constrained optimization problem:

$$
\begin{aligned}
\arg \min & \|\boldsymbol{\alpha}\|_{1}, \\
\text { s.t. } & \|\mathbf{y}-\boldsymbol{\Phi} \mathbf{x}\|<\eta,
\end{aligned}
$$

where $\eta$ is a preset threshold and $\|\boldsymbol{\alpha}\|_{1}$ is the $\ell_{1}$ norm of $\boldsymbol{\alpha}$. The above problem formulation leads to various CS reconstruction algorithms, such as Compressive Sensing Matching Pursuit (CoSaMP), Orthogonal Matching Pursuit (OMP), $\ell_{1}$ magic [20], and Lasso.

2.3. 1-Bit Compressive Sensing. Unlike conventional CS, 1-bit compressive sensing only keeps the sign bit of measurements and discards the magnitude information [10]. In matrix form,

$$
\mathbf{y}=\operatorname{sign}(\boldsymbol{\Phi} \mathbf{x}) \text {. }
$$

While traditional CS methods use equation constraint, 1bit CS reconstruction can be accomplished via optimization problem with an inequality constraint:

$$
\widehat{\mathbf{y}} \Phi \mathbf{x} \geq \mathbf{0},
$$

where $\widehat{\mathbf{y}}=\operatorname{diag}(\mathbf{y})$ and the inequality is applied for each component of the vector. Thus, the 1-bit CS reconstruction optimization problem can be expressed as follows:

$$
\begin{aligned}
\arg \min & \|\boldsymbol{\alpha}\|_{1} \\
\text { s.t. } & \mathbf{Y} \boldsymbol{\Phi} \Psi \mathbf{x} \geq 0, \\
& \|\boldsymbol{\alpha}\|_{2}=1 .
\end{aligned}
$$

The $\ell_{1}$ norm is used as a cost function that enforces the sparseness of $\boldsymbol{\alpha}$ under the sign constraints. Since the amplitude information is discarded in 1-bit sampling, a unit sphere constraint is introduced to avoid getting the obvious wrong solution of $\boldsymbol{\alpha}=\mathbf{0}$.

Several algorithms have been proposed to solve the 1bit CS reconstruction problem. These include 1-bit Linear Programming (1-bit LP), Matching Sign Pursuit (MSP) algorithm, Binary Iterative Hard Thresholding (BIHT), Restricted-Step Shrinkage (RSS), and Fixed Point Continuation (FPC).

2.4. Multiple Measurement Vectors. For multiple sparse signal with the same sparse pattern, the multiple measurement vectors (MMV) [21] formulation utilizes the joint sparse in reconstruction procedure. The MMV problem can be formulated as solving multiple underdetermined systems of linear equations:

$$
\mathbf{Y}=\boldsymbol{\Phi} \mathbf{A},
$$

where $\Phi$ is a full rank $M \times N$ matrix $(M \ll N)$ and $\mathbf{Y}$ is $M \times L$ observation matrix consisting of $L$ consecutive observations of $L$ sparse vector represented by $\mathbf{A}$. It is further assumed that each column of the A matrix has the same sparse structure.

Lots of algorithms have been proposed that exploit the joint sparsity in different ways. These include Simultaneous Orthogonal Matching Pursuit (SOMP), Reduce MMV and Boost (ReMBo), $\ell_{1}$-svd [12], and Multiple FOCal Underdetermined (M-FOCUSS) algorithms.

\section{Compressed Sampling}

In this section, we provide two sampling methods designed for WSAN. We try to reduce the transmission data volume for array signal, which brings energy consumption and transmission delay issues. Compared with traditional data compression methods, CS performs dimension reduction with projection calculation. Further comparisons will be provided in later sections.

3.1. Random Compressed Sampling. Random projection is an important step in compressive sensing methods. But random number generation and projection calculation are required to perform random projection on nodes. These raise energy consumption and hardware requirement. Inspired by nonuniform sampling method, we combine the nonuniform sampling process with CS framework and model it by a measurement matrix. It is easy to implement and no extra computation is needed on wireless nodes.

So the measurement matrix is designed as follows: the $M \times N$ measurement matrix $\Phi$ represents the measurement process; each row has only one nonzero component 1 in the $\left(m, t_{m}\right)$ position. Following this matrix, the ADC take the $m_{\text {th }}$ samples at $t_{m}$. A sequence $\mathbf{u}=\{u(1), u(2), \ldots, u(M)\}$ is set with $u(1)=1$, and

$$
u(m)=u(m-1)+\left[\tau_{m}\right], \quad 2 \leq m \leq M,
$$

where $\tau_{m} \in N\left(N / M, M^{2} K^{2} / N^{2}\right)$ is the random sampling interval between adjacent sample instances. Then the $(m, n)$ th element of this proposed random sampling matrix is given by

$$
\Phi(m, n)= \begin{cases}1 & 1 \leq n=\mathbf{u}(m) \leq N ; \\ 0 & \text { otherwise. }\end{cases}
$$

And this $\boldsymbol{\Phi}$ is used as the measurement matrix in the random compressed sampling method. Although we call it random, this matrix (or sampling sequence) could be predefined and saved in nodes and fusion center. Nodes do not have to generate random number themselves.

3.2. 1-Bit Compressed Sampling. 1-bit compressed sampling has its obvious advantage in reducing data volume. On condition that the sampling rate can be supported in sensor node, 1-bit compressed sampling will be an appealing solution to WSAN monitoring applications. And there are two ways of implementation on nodes.

(1) Hardware Implementation. Use random number generators and comparators on nodes. After random projection of raw signal, a comparator to zero is utilized to quantize the measurement. And, in fact, this kind of comparators is extremely cheap and fast.

(2) Software Implementation. Perform random projection in software. The ADC samples at the Nyquist rate and only the signs of projected raw samples are kept. Such method has been successfully used in 1-bit SigmaDelta converters, at the expense of very high sampling rate [22]. 
3.3. The Coherence of Compressed Sampling. To use CSbased methods, the incoherence of measurement matrix and sparsity matrix should be tested. Note that the structure of $\boldsymbol{\Phi}$ and the product $\boldsymbol{\Phi} \cdot \boldsymbol{\Psi}$ amount to selecting some rows from the Discrete Fourier Transform (DFT) matrix $\Psi$. Hence their product $\boldsymbol{\Phi} \cdot \Psi$ is a partial Fourier transform matrix (submatrix of full DFT matrix). For the underlying random nonuniform sampling, the selection of these $M$ rows is determined by u. Applying the Welch bound inequality [23], when $\mathbf{u}$ is randomly selected from $\{1,2, \ldots, N\}, \mu$ satisfies

$$
\mu(\Phi \Psi) \leq \sqrt{\frac{N-M}{(N-1) M}} .
$$

For 1-bit CS, binary $\varepsilon$-stable embedding $(\mathrm{B} \varepsilon \mathrm{SE})$ is proposed to characterize 1-bit CS system [24], and robust reconstruction is guaranteed when $M>C_{\varepsilon} K \log N$.

\section{Collaborative Reconstruction}

Conventional CS methods guarantee stable signal recovery with the prior information of sparsity. However, the sparsity is not the only prior information that helps to recover signal in this sensor array case. For spatially neighboring nodes in the same array, they receive highly correlated signals. The correlations are displayed in their similarity in spectrum, although different in phase shift. (They share the same support in frequency domain.) Thus independent reconstruction of individual sensor signal seems redundant.

4.1. Reconstruction for Random Compressed Sampling. Similar to the MMV formulation, the compressed measurements of all $J$ sensors can be formulated as

$$
\mathrm{Y}=\boldsymbol{\Phi X}=\Phi \Psi \mathrm{A},
$$

where $\mathbf{Y}=\left[\mathbf{y}_{1}, \mathbf{y}_{2}, \ldots, \mathbf{y}_{J}\right], \mathbf{X}=\left[\mathbf{x}_{1}, \mathbf{x}_{2}, \ldots, \mathbf{x}_{J}\right]$, and $\mathbf{A}=\left[\boldsymbol{\alpha}_{1}, \boldsymbol{\alpha}_{2}, \ldots, \boldsymbol{\alpha}_{J}\right]$. Note that each $\mathbf{x}_{j}$ is a summation of different delay versions of sources signal; $\boldsymbol{\alpha}_{j}$ has the same sparse pattern. Based on such joint sparsity, the signal reconstruction problem can be described as

$$
\begin{array}{ll}
\arg \min _{\mathbf{A}} & \|\mathbf{d}\|_{1} \\
\text { s.t. } & \|\mathbf{Y}-\boldsymbol{\Phi} \mathbf{\Psi} \mathbf{A}\|_{2} \leq \sigma, \\
& \mathbf{d}(n)=\sum_{j=1}^{J} \alpha_{j}^{2}(n) .
\end{array}
$$

Under this joint reconstruction model, the theoretical measurement number of random compressed sampling is $L K+$ $\log (N / K)$ [25] while the normal CS requires $c J K \log (N / K)$ [8]. The computational complexity of the above joint reconstruction is $\mathscr{O}\left(J^{3} N^{3}\right)$ [21]; it sacrifices the real-time performance of signal reconstruction.

To solve this issue, we propose a collaborative signal reconstruction method. The main idea of this proposed framework is to choose one reference sensor node and reconstruct its signal. Then the support (nonzeros index of a sparse vector) of the reconstructed signal can be utilized to prune the CS problem to a least square problem.

We define $\boldsymbol{\Omega}=\operatorname{supp}(\widehat{\boldsymbol{\alpha}})$ to be the support of reference signal. Based on the known support $\Omega$, the joint reconstruction formulation can be simplified to

$$
\mathbf{Y}=\boldsymbol{\Theta}_{\Omega} \mathbf{A}_{\Omega}
$$

where $\boldsymbol{\Theta}_{\Omega}$ and $\mathbf{A}_{\Omega}$ are the submatrices of $\boldsymbol{\Theta}$ and $\mathbf{A}$ with column and row index $\Omega$.

It is obvious that $\boldsymbol{\Theta}_{\Omega}$ and $\mathbf{A}_{\Omega}$ are $M \times T$ and $T \times J$ matrices $(T=|\Omega|)$. In such case, the traditional ill-determined optimization problem is equivalent to solving equations with $M$ measurement and $T$ unknown variable. When $M>T$, it has a least squares solution:

$$
\mathbf{A}_{\Omega}=\left(\boldsymbol{\Theta}_{\Omega}^{T} \boldsymbol{\Theta}_{\Omega}\right)^{-1} \boldsymbol{\Theta}_{\Omega}^{T} \mathbf{Y}
$$

In conclusion, when given the support of signal, the signal reconstruction problem is simplified to a least square problem, which means we only need $T$ measurements to solve it. The pseudocode of collaborative reconstruction is presented as follows. ( $\ell_{1}$-magic [20] is used as an example.)

Algorithm 1 (collaborative reconstruction algorithm).

Input

Compressed sampling matrix $\boldsymbol{\Phi}$;

Measurements $\mathbf{y}_{j}(j=1,2, \ldots, J)$.

Output

K-sparse approximation $\boldsymbol{\alpha}_{j}(j=1,2, \ldots, J)$.

(1) Solve $\boldsymbol{\alpha}_{1}$ by $\ell_{1}$-magic algorithm:

$$
\begin{aligned}
\arg \min & \left\|\boldsymbol{\alpha}_{1}\right\|_{1} \\
\text { s.t. } & \mathbf{y}_{1}=\boldsymbol{\Phi} \Psi \boldsymbol{\alpha}_{1} .
\end{aligned}
$$

(2) Estimate the support of $\boldsymbol{\alpha}_{1}$ :

$$
\operatorname{supp}\left(\boldsymbol{\alpha}_{1}\right)=\left\{n \mid \boldsymbol{\alpha}_{1}(n)>\varepsilon\right\}
$$

(3) Obtain the pruned reconstruction matrix:

$$
\boldsymbol{\Theta}_{\Omega} \longleftarrow\left(\boldsymbol{\Theta}, \operatorname{supp}\left(\boldsymbol{\alpha}_{1}\right)\right)
$$

(4) Analytical solution of a least square problem:

$$
\boldsymbol{\alpha}_{j}=\left(\boldsymbol{\Theta}_{\Omega}^{T} \boldsymbol{\Theta}_{\Omega}\right)^{-1} \boldsymbol{\Theta}_{\Omega}^{T} \mathbf{y}_{j} \quad(j=2,3, \ldots, J) .
$$

4.2. Collaborative Reconstruction for 1-Bit Sampling. For 1-bit compressed sampling, whose measurements are the sign of projection for the original signal, the nonlinearity between the original signal and the measurements makes it hard to find an analytical solution. However, the obtained support can still help to simplify and boost the reconstruction process. 
Similar to the reconstruction for random compressed sampling, the 1-bit reconstruction problem can be modeled as

$$
\begin{array}{ll}
\min & \sum_{i=1}^{N} u_{i} \\
\text { s.t. } & -u_{i} \leq \alpha_{i} \leq u_{i}, \quad i=1,2, \ldots, N, \\
& y_{i}\left(\boldsymbol{\theta}_{i}^{T} \boldsymbol{\alpha}\right) \geq 0, \quad i=1,2, \ldots, M, \\
& \sum_{i=1}^{M} y_{i}\left(\boldsymbol{\theta}_{i}^{T} \boldsymbol{\alpha}\right) \geq M, \quad i=1,2, \ldots, M, \\
& \alpha_{i}=0, \quad i \notin \operatorname{supp}\left(\boldsymbol{\alpha}_{0}\right),
\end{array}
$$

where $\boldsymbol{\theta}$ is the $i$ th column of $\boldsymbol{\Theta}$.

For both sampling methods above, collaborative reconstruction algorithm simplifies the redundant reconstruction dictionary with the help of signal support. It avoids the redundancy in traditional CS signal reconstruction. Therefore, this algorithm can greatly reduce the computational complexity and increase the success rate of reconstruction while requiring less information. These merits have great value in achieving low-power, real-time wireless sensing systems.

\section{Performance Evaluation}

In this section, extensive simulations are carried out to compare the proposed framework with traditional array processing and CS approaches. Consider the modern data compression approach and the wireless channel that has transmission error; our framework is compared with some local data compression approach under different transmission error cases. Finally, a hardware prototype is developed to validate the practical application of the framework through outdoor experiments.

5.1. Experiment and Simulation Settings. Theoretically, our framework works for any array formation when it satisfies the array spacing requirements. Here, a uniform linear array with 6 nodes and $\mathrm{a}-90^{\circ}$ to $90^{\circ}$ target space is chosen for both simulation and experiment. Considering that the acoustic signals of cars, trucks, or helicopters are usually dominated by a few harmonics, the source signal is assumed to be summation of harmonics at $500 \mathrm{~Hz}, 600 \mathrm{~Hz}, 700 \mathrm{~Hz}$, and $800 \mathrm{~Hz}$. Accordingly, a $0.2 \mathrm{~m}$ spacing is adopted to satisfy the half-wavelength requirements of array.

We choose the widely accepted MUSIC [16] algorithm for DoA estimation. The dimension of original signal (also reconstructed signal) in a snapshot is assumed to be 128 , and the system sampling rate is set to be $2048 \mathrm{~Hz}$. For expression simplicity in this section, we denote RCS as random compressed sampling, $1 \mathrm{bS}$ as 1-bit compressed sampling, and CoR as collaborative reconstruction. The joint reconstruction method mentioned in Section 4 is used as benchmark while evaluating the proposed CoR method, denoted as JoR. To have fair comparison, we formulate the random compressed
TABLE 1: Measurement number settings for reconstruction.

\begin{tabular}{lc}
\hline Method & Samples \\
\hline Raw signal & 128 for all nodes \\
RCSJoR & 32 for all nodes \\
RCSCoR & 64 for ref node, 16 for others \\
bSCoR & 178 bits for ref node, 64 bits for others \\
\hline
\end{tabular}

sampling based approach as an SOCP problem and 1-bit CS base approach as an LP optimization. Both SOCP and LP are solved by Sedumi toolbox [26].

The first problem of CS in real application is the number of random measurements. Although some theoretic bounds have been proposed, it is still not clear how many samples will bring maximum benefit. Here the SNR of reconstructed signal is chosen as criteria. The SNR of raw signal is $10 \mathrm{~dB}$ and the reconstructed signals are compared with uncontaminated signal to obtain the same SNR. Figure 3 shows the relationship between the reconstructed SNR and the corresponding measurements $M$. Table 1 shows the number of measurements necessary for a method to reach original $10 \mathrm{~dB}$ SNR.

As we can see here, CS-based methods obviously reduce the data volume, while our RCSCoR and 1bSCoR methods further reduce the number of measurements for nonreference nodes.

5.2. DoA Estimation Comparison. Figure 4 displays the DoA estimation result of three CS-based approaches using above empirical measurements numbers, and the RS (raw signal) denotes array processing with raw data, used as benchmark. First, we use settings from Table 1 for all methods. Results indicate that all methods present spatial spectrum clear enough to tell the source direction. RS has the best performance. For compressed sampling methods, reduction in sample amount caused information loss; therefore they all present worse performance but still clear directions.

Further numerical simulations are performed to statistically show the performances of these CS-based frameworks for DoA estimation purpose. The root mean square error (RMSE) and success detection rate of 500 independent trials are used as performance metrics. Here, if a DoA estimation result falls in $\pm 10^{\circ}$ range of true value, it is viewed as a success.

From the results in Figure 5, we could tell that using full raw signal simply provides the best performances. That makes sense for no information loss introduced. Therefore, the RS results may be considered as true value or benchmark of estimation performances. Other three CS-based methods are inferior to the RS approach, but still in a tolerable range. Among these three CS-based approaches, RCSJoR outperforms RCSCoR and lbSCoR. This is because the RCSJoR approach is based on a joint optimization while others rely on the sparsity of single sensor.

In the above tests, we used settings from Table 1. These settings come from SNR analysis of reconstructed signal. The difference of these CS-based approaches in RMSE and success rate indicates that the criterion of reconstructed SNR may not be the most suitable one. Here, in Figure 6, the RMSE of 

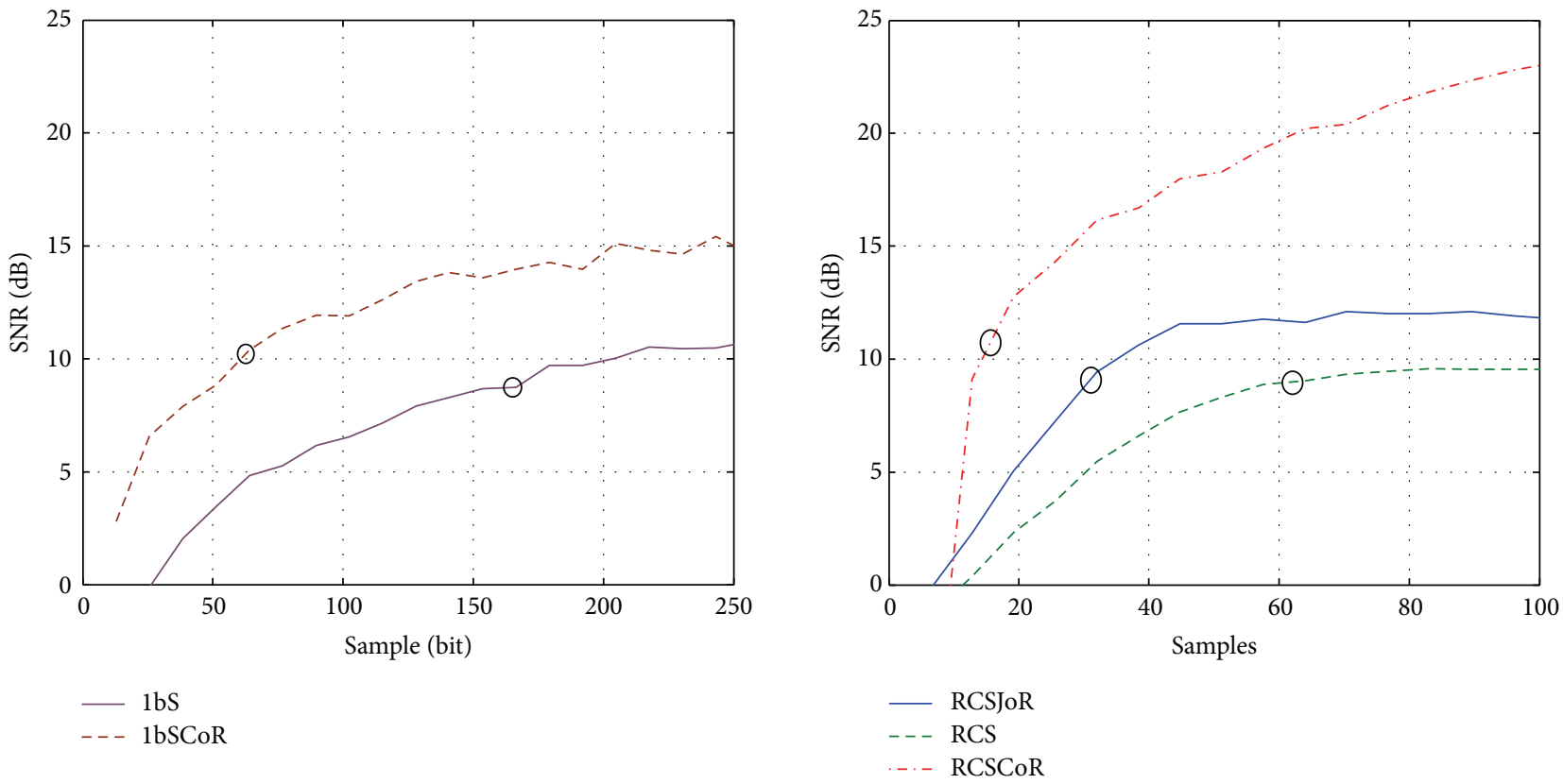

FIgURE 3: Comparison of measurement number $M$.

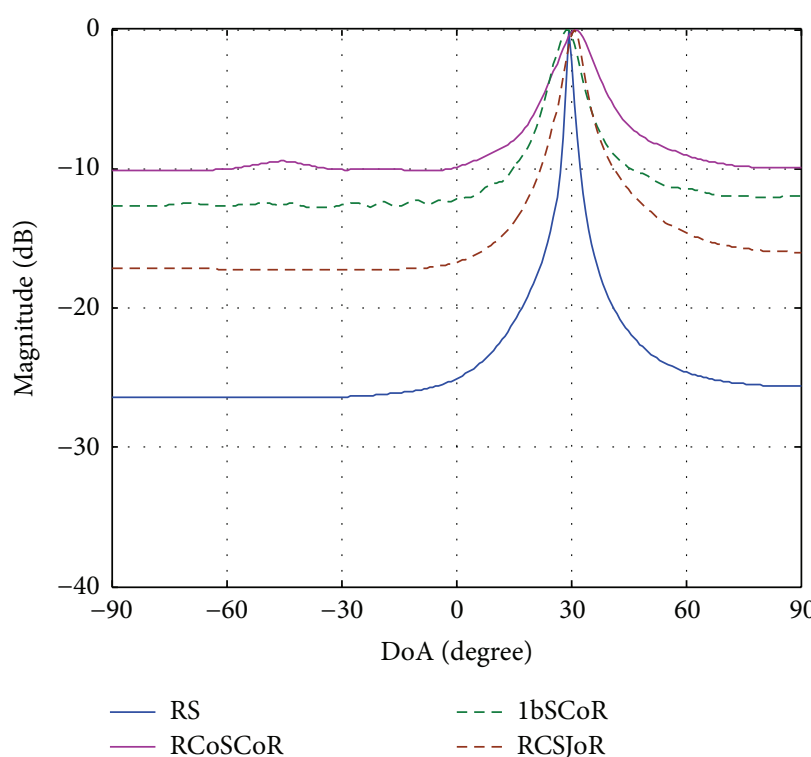

(a)

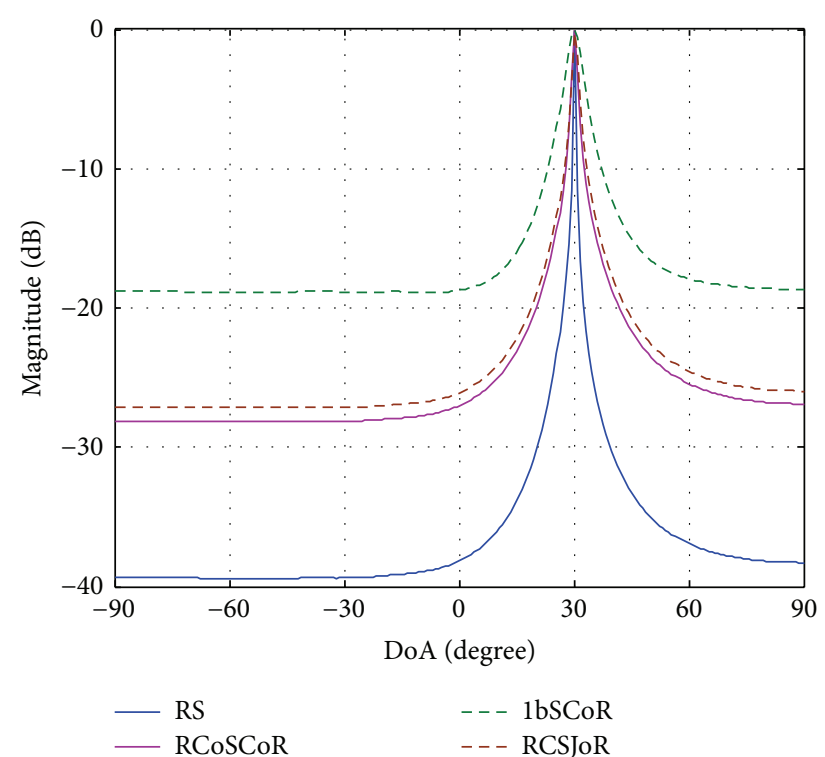

(b)

FIgURE 4: MUSIC results under SNR $5 \mathrm{~dB}$ (a) and $20 \mathrm{~dB}$ (b).

DoA is chosen as criteria that evaluate different measurement number $M$. To be fair, three times the RMSE value of RS approach is used as performance degradation threshold for each method and each SNR level (presented by dotted lines with corresponding color in Figure 6). This threshold may not be optimum but should provide credible results. Based on such threshold, we choose settings that could guarantee most SNR cases for each method. Then we have $M=21$ for RCSCoR, $M=15$ for RCSJoR, and $M=120$ for $1 b S C o R$.
These settings are for nonreference nodes. Reference nodes still use Table 1 setting.

5.3. Compression Comparison. CS-based methods could also be considered as special-purpose lossy compression methods. Ignoring algorithm complexity problem, other local data compression approaches can also reduce the data volume before wireless transmission. So here, we compare the performance of CS-based methods with local data compression 


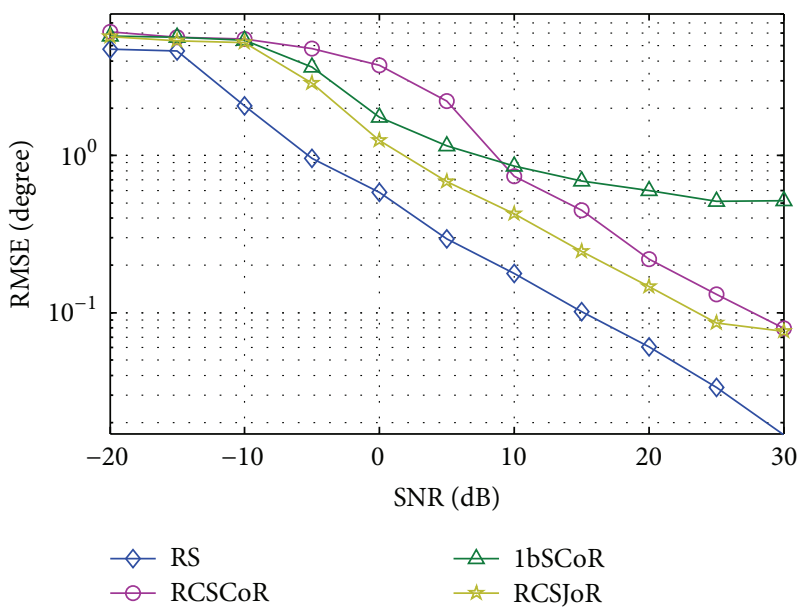

(a)

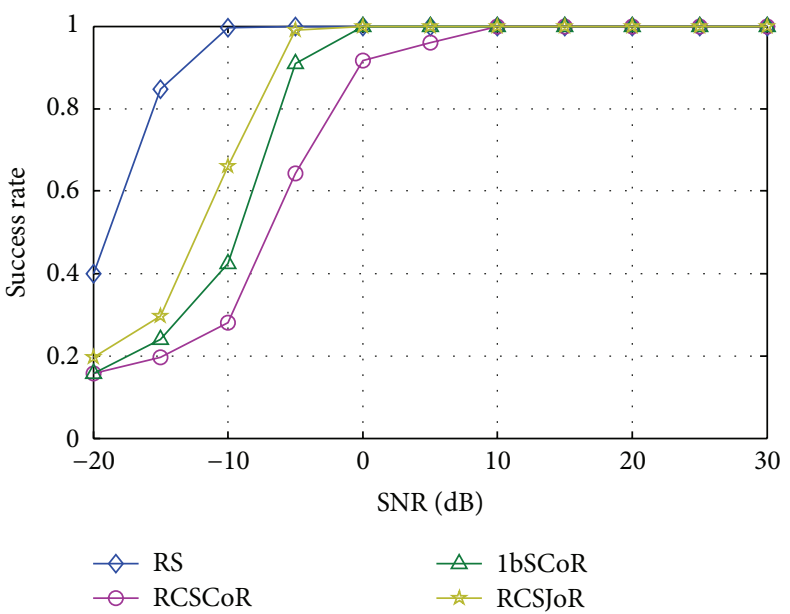

(b)

Figure 5: DoA RMSE (a) and success rate (b) against SNR.

RCSCoR

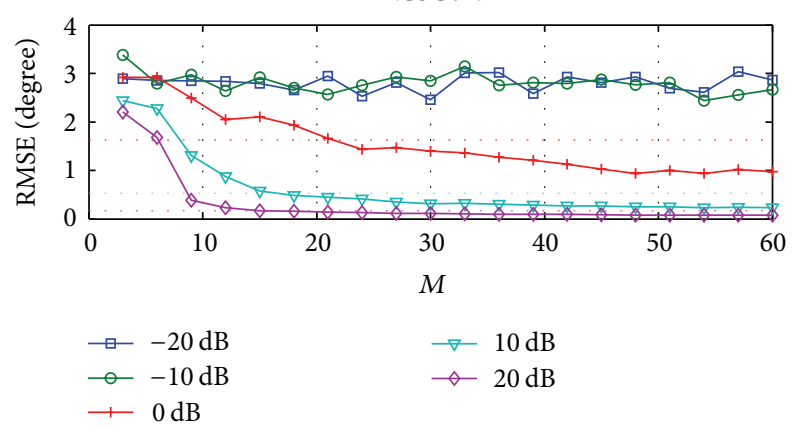

RCSJoR

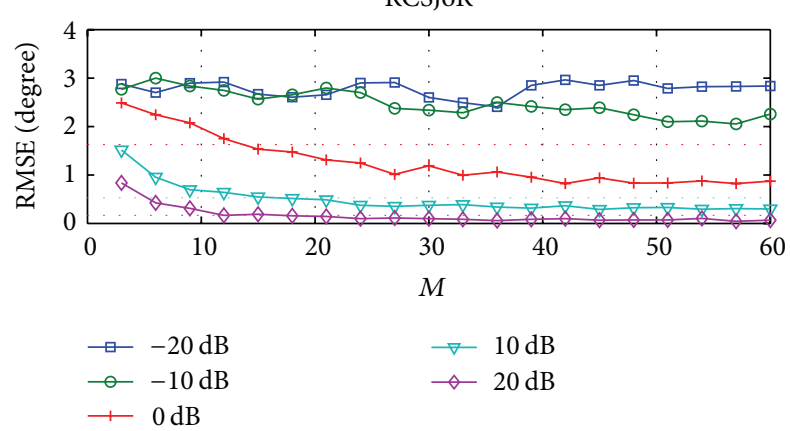

lbSCoR

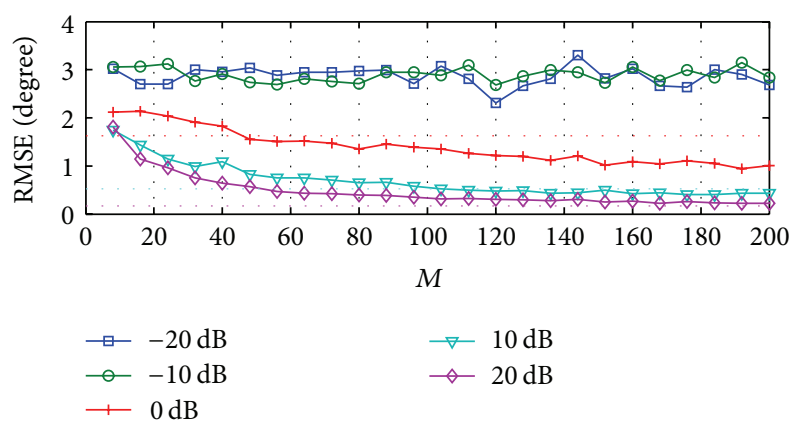

FIGURE 6: DoA performance of three methods against $M$.
TABLE 2: Compression ratio of all methods.

\begin{tabular}{lcccccc}
\hline Method & RCSCoR & RCSJoR & lbSCoR & LZW & JPEG & MPEG-1 \\
\hline Ratio & 4.544 & 8.533 & 15.794 & 1.184 & 3.103 & 1.871 \\
\hline
\end{tabular}

methods. Three typical data compression methods are considered: the lossless weighted LZW coding [17], lossy JPEG [18], and MPEG audio coding layer 1 [19].

Referring to the technical manual of STM32 series MCU, the validated package load is 100 bytes and the PER (Package Error Rate) is 1\% with package of 20 bytes. Based on the provided parameter, the BER (Bit Error Rate) is set to be $6.28 \times 10^{-5}, 1.26 \times 10^{-4}, 3.21 \times 10^{-4}$, and $6.58 \times 10^{-4}$ (corresponding to PER of $1 \%, 2 \%, 5 \%$, and $10 \%$, resp.). Here, we use the $M$ values mentioned in previous section, namely, 21 for RCSCoR, 15 for RCSJoR, and 120 bits for 1 bSCoR. For methods other than $1 \mathrm{bS}$, we normally use 16-bit quantization in following computation.

Figure 7 shows DoA success rate of different BERs, and RMSE are presented as error bars. Obviously, CS-based methods display robust property under all the BERs, while not all existing compression methods can. The LZW has an obvious performance decline when the BER increases; the JPEG compression algorithm is heavily affected by the BERs; only MPEG-1 displays a strong error tolerant capability, but not better than CS-based approaches. This is because the CSbased approaches focus on the sparse structure of data and the error of transmission can be regarded as noise and hence eliminated through reconstruction algorithms.

Table 2 lists the compression ratio of CS-based and traditional data compression approaches. The compression ratio for CS-based approaches is much high than that of the conventional compression approaches. Here, LZW has the smallest compression ratio; this may be because the repeatability of data is not good enough and extra data volume is occupied to construct coding dictionary. For different cases, CS-based methods could work with different parameter 

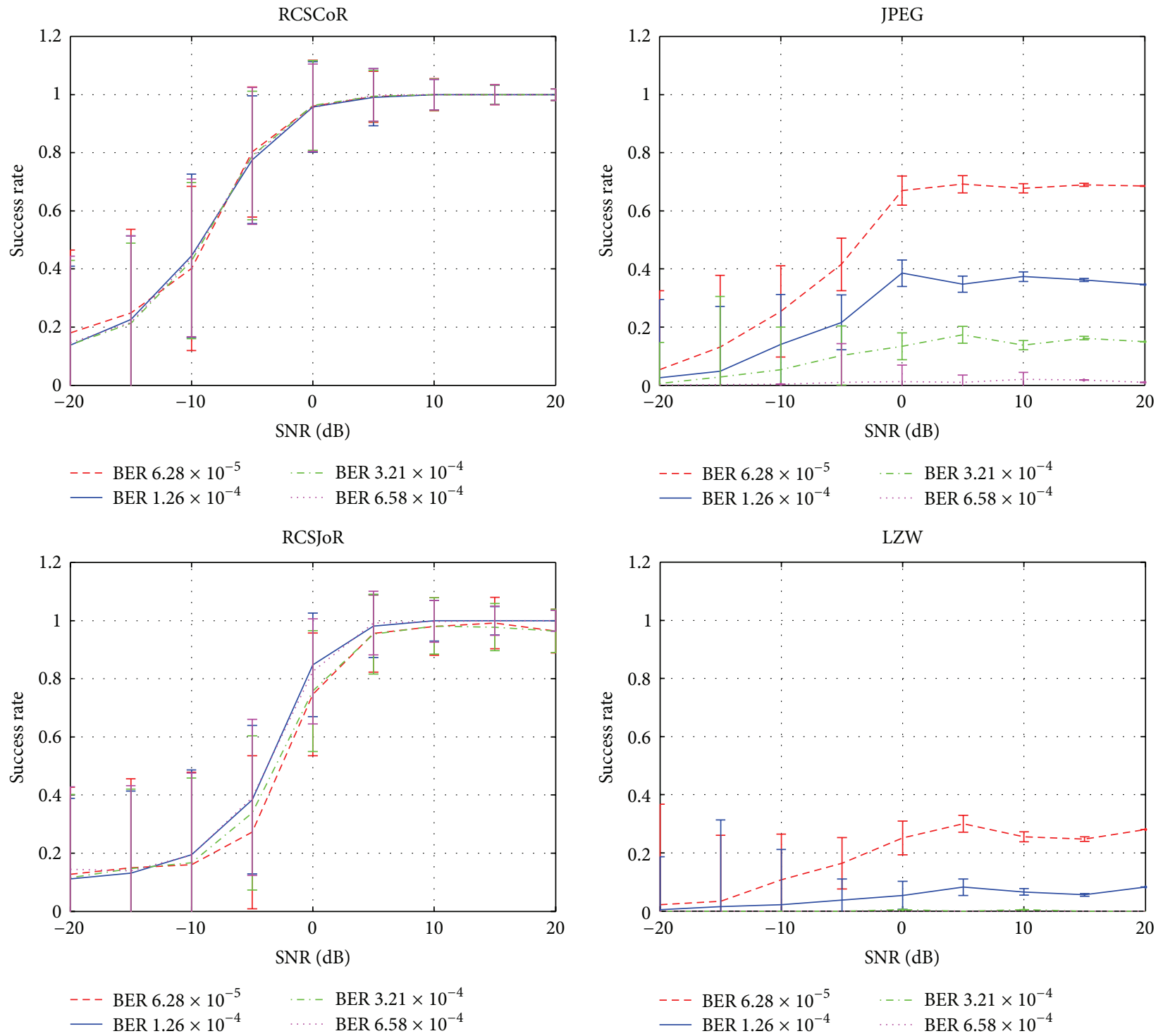

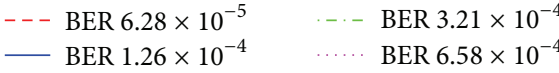

IbSCoR
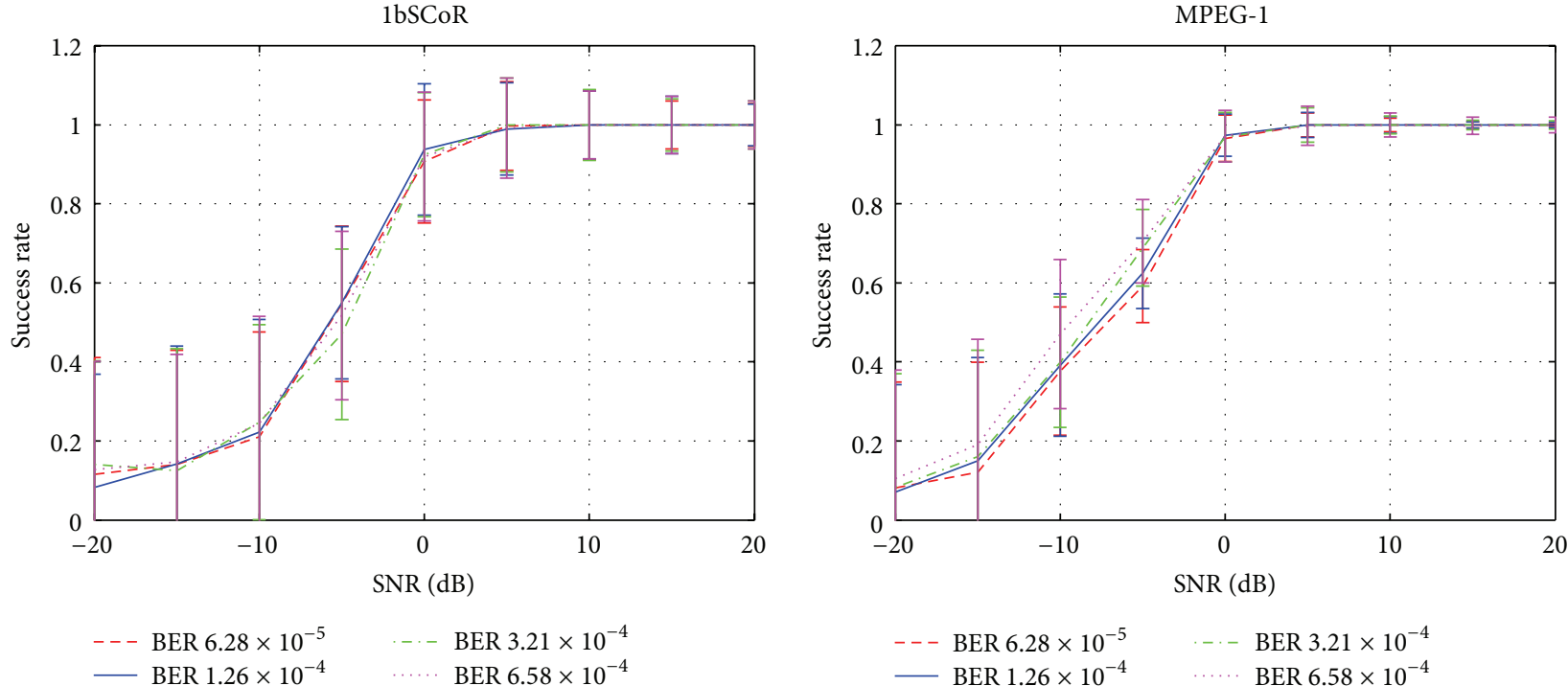

Figure 7: DoA comparison under different BERs. 


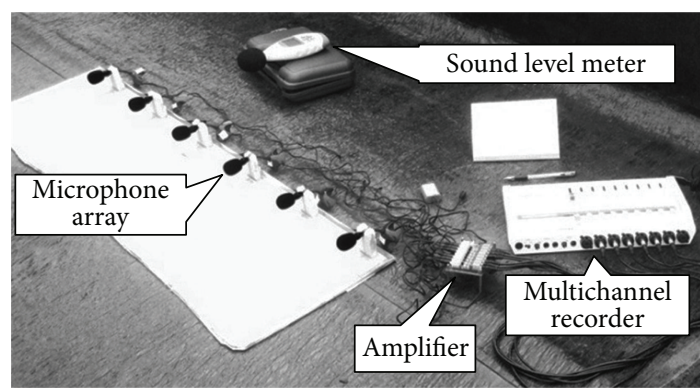

FIGURE 8: Prototype system in experiments.

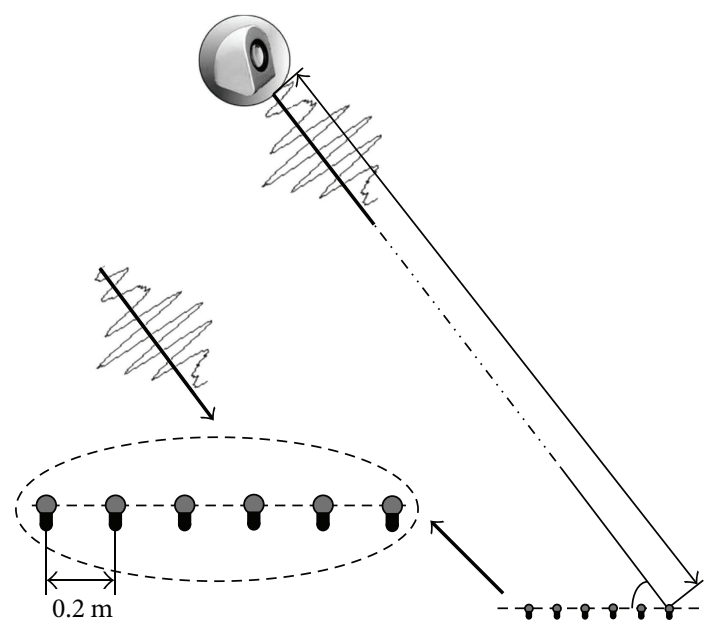

FIGURE 9: Experiment setting.

settings. This flexibility offers them better adaptability for various cases.

5.4. Field Experiment. To validate the proposed framework, an acoustic sensor array prototype system is developed to conduct some field experiments. As shown in Figure 8, this prototype mainly consists of a uniformly spaced linear array with six omnidirectional microphones and a multichannel recorder. Spacing between adjacent microphones is $0.2 \mathrm{~m}$. Figure 9 shows the experiment setting. One source is placed at direction $60.2^{\circ}$, few metres away. The sampling frequency is $2048 \mathrm{~Hz}$ and the DoA is estimated every 3 snapshots (each snapshot with 128 samples).

Figure 10 shows the spatial spectrum of CS-based approaches and also RS as benchmark. The DoA results are $57.9^{\circ}$ for $1 \mathrm{bSCoR}, 58.1^{\circ}$ for RCSCoR, $58.1^{\circ}$ for RCSJoR, and $58.25^{\circ}$ for RS. This result shows that our framework could work well on real off-the-shelf devices.

5.5. Performance Summary. Till now, with simulation and experiment results, we can reach the following conclusions. (a) The proposed framework could significantly reduce sample data volume with a little performance loss; (b) RCSCoR could work with extremely simple nodes while $1 \mathrm{bSCoR}$

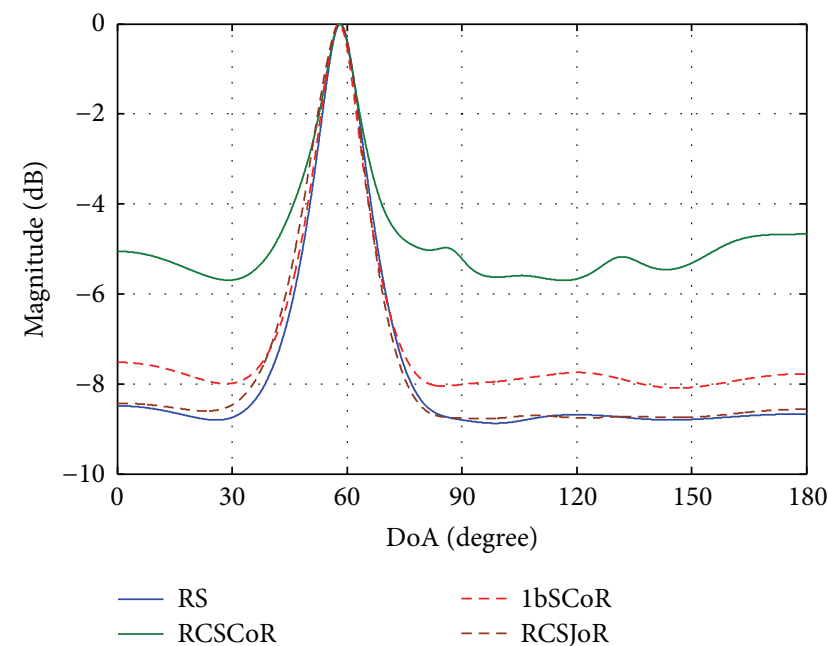

FIGURE 10: MUSIC results of prototype system.

provide best DoA performance with least data. Which to use depends on the systems requirements.

\section{Conclusion}

In this paper, we propose a compressed sampling and collaborative reconstruction framework. By exploiting the sparse property of source signal, random compressed sampling and 1-bit sampling require much less array data while still providing acceptable performance. By exploiting the high correlation among array signals, collaborative reconstruction effectively reconstructs array signal as well as further reducing the number of samples required for nonreference nodes.

Different methods have different advantages. RCSCoR method could work well with simple devices, and 1bSCoR provides the highest compression ratio. Both numerical simulation and experiments on off-the-shelf device are presented to validate the usefulness of the proposed framework. Considering the convenience of implementation on hardware, this framework would be an excellent choice on low-cost, low-power wireless sensor array network.

\section{Competing Interests}

The authors declare that there is no conflict of interests regarding the publication of this paper.

\section{Acknowledgments}

This work was supported in part by the National Natural Science Foundation of China (NSFC61273079), in part by the National Natural Science Foundation of China Key Projects (U1509215), in part by the Strategic Priority Research Program of the Chinese Academy of Sciences (Grant no. XDA06020201), and in part by the Open Research Project of the State Key Laboratory of Industrial Control Technology, Zhejiang University (no. ICT1600208, no. ICT1600199, and no. ICT1600213). 


\section{References}

[1] A. M. Ali, S. Asgari, T. C. Collier et al., "An empirical study of collaborative acoustic source localization," Journal of Signal Processing Systems, vol. 57, no. 3, pp. 415-436, 2009.

[2] A. Ledeczi, A. Nadas, P. Volgyesi et al., "Countersniper system for urban warfare," ACM Transactions on Sensor Networks, vol. 1, no. 2, pp. 153-177, 2005.

[3] Y. Li, Z. Wang, S. Zhuo et al., "The design and implement of acoustic array sensor network platform for online multitarget tracking," in Proceedings of the 8th IEEE International Conference on Distributed Computing in Sensor Systems (DCOSS '12), pp. 323-328, IEEE, Hangzhou, China, May 2012.

[4] J. C. Chen, K. Yao, T. L. Tung, C. W. Reed, and D. Chen, "Source localization and tracking of a wideband source using a randomly distributed beamforming sensor array," International Journal of High Performance Computing Applications, vol. 16, no. 3, pp. 259-272, 2002.

[5] J. C. Chen, L. Yip, J. Elson et al., "Coherent acoustic array processing and localization on wireless sensor networks," Proceedings of the IEEE, vol. 91, no. 8, pp. 1154-1161, 2003.

[6] M. Allen, L. Girod, R. Newton, S. Madden, D. T. Blumstein, and D. Estrin, "VoxNet: an interactive, rapidly-deployable acoustic monitoring platform," in Proceedings of the International Conference on Information Processing in Sensor Networks (IPSN '08), pp. 371-382, St. Louis, Mo, USA, April 2008.

[7] W. Hu, N. Bulusu, C. T. Chou, S. Jha, A. Taylor, and V. N. Tran, "Design and evaluation of a hybrid sensor network for cane toad monitoring," ACM Transactions on Sensor Networks, vol. 5, no. 1, article 4, pp. 1-28, 2009.

[8] D. L. Donoho, "Compressed sensing," IEEE Transactions on Information Theory, vol. 52, no. 4, pp. 1289-1306, 2006.

[9] E. J. Candès, "The restricted isometry property and its implications for compressed sensing," Comptes Rendus Mathematique, vol. 346, no. 9-10, pp. 589-592, 2008.

[10] P. T. Boufounos and R. G. Baraniuk, "1-Bit compressive sensing," in Proceedings of the 42nd Annual Conference on Information Sciences and Systems (CISS '08), pp. 16-21, Princeton, NJ, USA, March 2008.

[11] P. T. Boufounos, "Reconstruction of sparse signals from distorted randomized measurements," in Proceedings of the IEEE International Conference on Acoustics, Speech, and Signal Processing (ICASSP '10), pp. 3998-4001, IEEE, Dallas, Tex, USA, March 2010.

[12] D. Malioutov, M. Cetin, and A. S. Willsky, "A sparse signal reconstruction perspective for source localization with sensor arrays," IEEE Transactions on Signal Processing, vol. 53, no. 8, pp. 3010-3022, 2005.

[13] D. Baron, M. B. Wakin, M. F. Duarte, S. Sarvotham, and R. G. Baraniuk, "Distributed compressed sensing," Tech. Rep., Department of Electrical and Computer Engineering, Rice University, Houston, Tex, USA, 2006.

[14] Z. Xiahou and X. Zhang, "Adaptive localization in wireless sensor network through Bayesian compressive sensing," International Journal of Distributed Sensor Networks, vol. 11, no. 8, Article ID 438638, 2015.

[15] T. L. N. Nguyen and Y. Shin, "Multiple target localization in wsns based on compressive sensing using deterministic sensing matrices," International Journal of Distributed Sensor Networks, vol. 2015, Article ID 947016, 8 pages, 2015.
[16] R. O. Schmidt, "Multiple emitter location and signal parameter estimation," IEEE Transactions on Antennas and Propagation, vol. 34, no. 3, pp. 276-280, 1986.

[17] M. R. Nelson, "LZW data compression," Dr. Dobb's Journal, vol. 14, no. 10, pp. 29-36, 1989.

[18] G. K. Wallace, "The JPEG still picture compression standard," Communications of the ACM, vol. 34, no. 4, pp. 30-44, 1991.

[19] K. Brandenburg and G. Stoll, "Iso/mpeg-1 audio: a generic standard for coding of high-quality digital audio," Journal of the Audio Engineering Society, vol. 42, no. 10, pp. 780-792, 1994.

[20] E. Candes and J. Romberg, " $l_{1}$-magic: recovery of sparse signals via convex programming," 2005.

[21] L. Sun, J. Liu, J. Chen, and J. Ye, "Efficient recovery of jointly sparse vectors," in Proceedings of the 23rd Annual Conference on Neural Information Processing Systems (NIPS '09), pp. 1812-1820, December 2009.

[22] P. M. Aziz, H. V. Sorensen, and J. Van der Spiegel, "An overview of sigma-delta converters," IEEE Signal Processing Magazine, vol. 13, no. 1, pp. 61-84, 1996.

[23] P. Xia, S. Zhou, and G. B. Giannakis, "Achieving the welch bound with difference sets," IEEE Transactions on Information Theory, vol. 51, no. 5, pp. 1900-1907, 2005.

[24] L. Jacques, J. N. Laska, P. T. Boufounos, and R. G. Baraniuk, "Robust 1-bit compressive sensing via binary stable embeddings of sparse vectors," IEEE Transactions on Information Theory, vol. 59, no. 4, pp. 2082-2102, 2013.

[25] R. G. Baraniuk, V. Cevher, M. F. Duarte, and C. Hegde, "Modelbased compressive sensing," IEEE Transactions on Information Theory, vol. 56, no. 4, pp. 1982-2001, 2010.

[26] Sedumi, http://sedumi.ie.lehigh.edu/. 


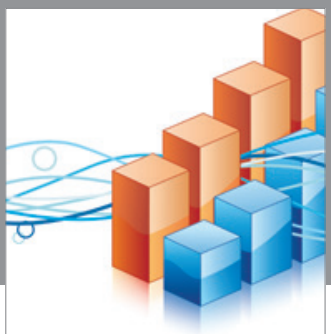

Advances in

Operations Research

vatem alat4

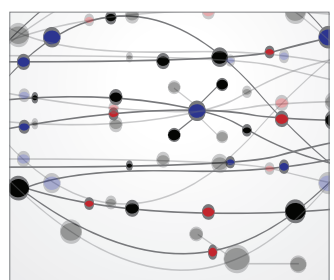

\section{The Scientific} World Journal
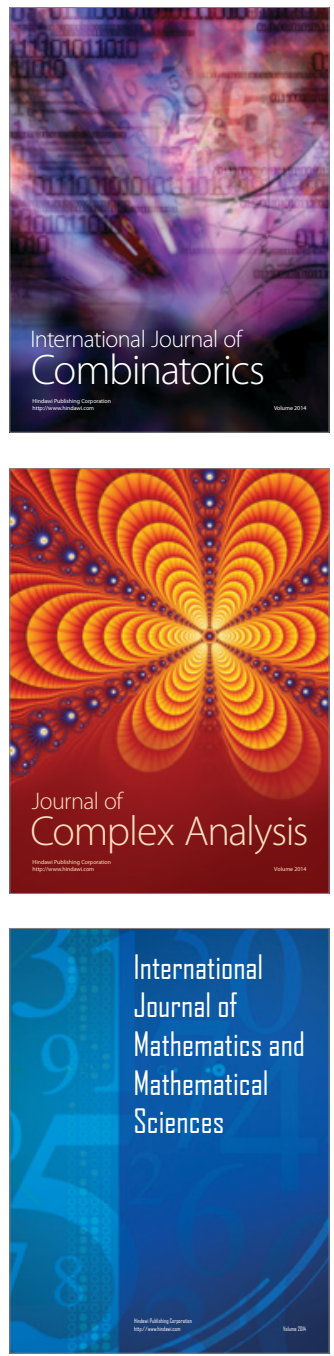
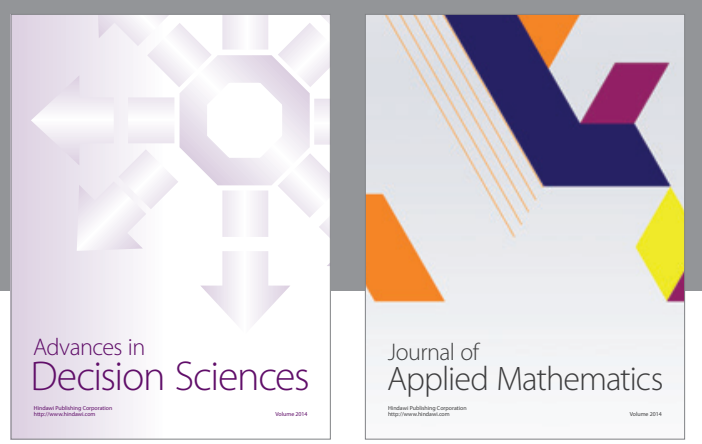

Algebra

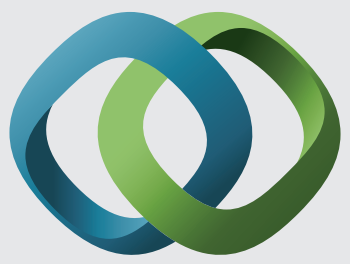

\section{Hindawi}

Submit your manuscripts at

http://www.hindawi.com
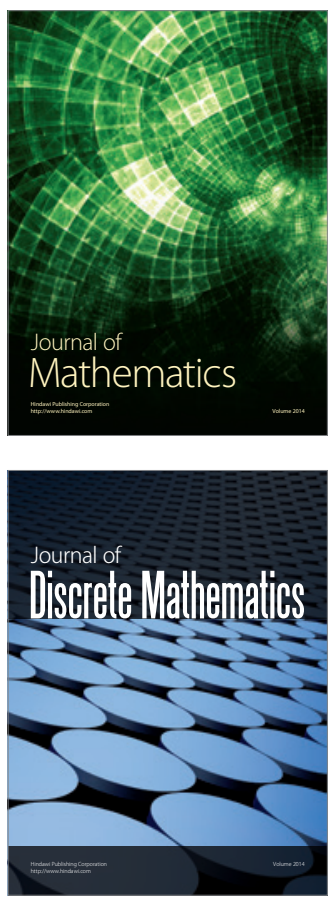

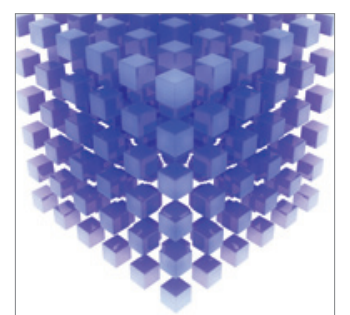

Mathematical Problems in Engineering
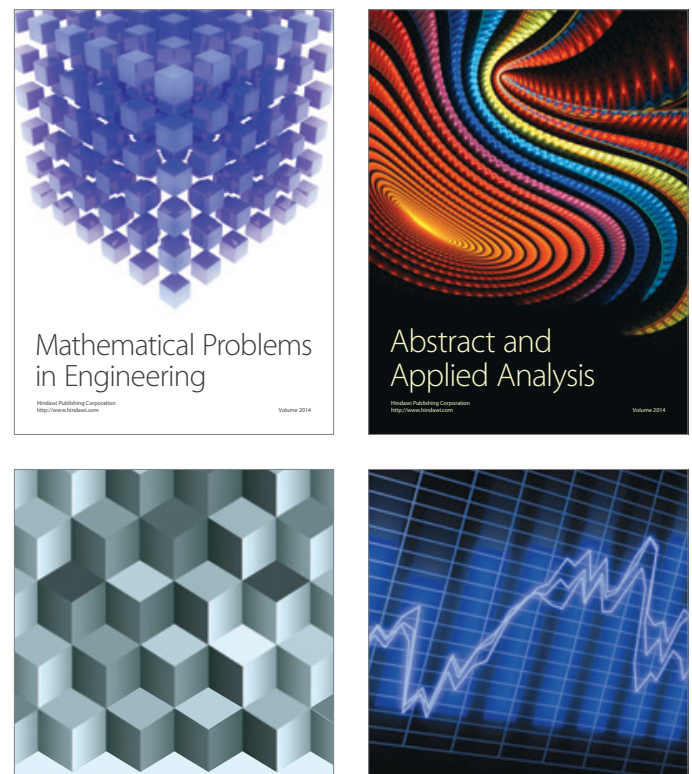

Journal of

Function Spaces

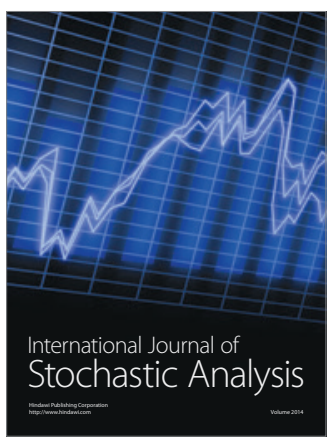

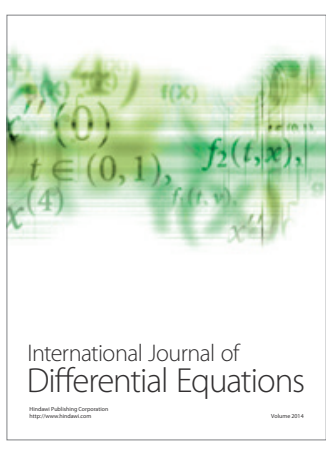
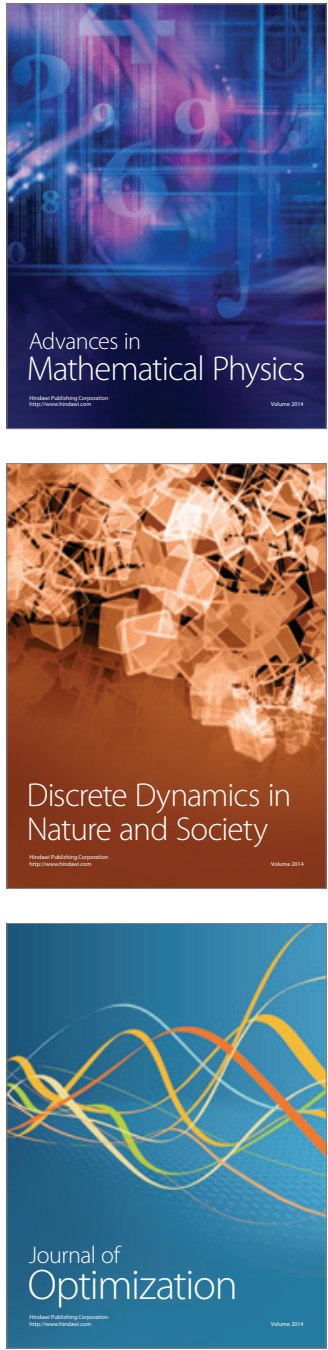